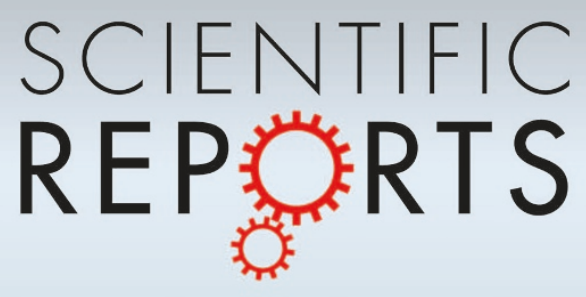

OPEN

SUBJECT AREAS:

METAMATERIALS

LITHOGRAPHY

TWO-DIMENSIONAL MATERIALS

SURFACE PATTERNING

Received

20 August 2013

Accepted

11 November 2013

Published

28 November 2013

Correspondence and requests for materials should be addressed to

Y.-C.C. lychang6@ mail.ncku.edu.tw)

\section{High-Throughput Nanofabrication of Infra-red and Chiral Metamaterials using Nanospherical-Lens Lithography}

\author{
Yun-Chorng Chang, Sih-Chen Lu, Hsin-Chan Chung, Shih-Ming Wang, Tzung-Da Tsai \& Tzung-Fang Guo
}

Department of Photonics and Advanced Optoelectronic Technology Center, National Cheng Kung University, Tainan 701, Taiwan.

Various infra-red and planar chiral metamaterials were fabricated using the modified Nanospherical-Lens Lithography. By replacing the light source with a hand-held ultraviolet lamp, its asymmetric light emission pattern produces the elliptical-shaped photoresist holes after passing through the spheres. The long axis of the ellipse is parallel to the lamp direction. The fabricated ellipse arrays exhibit localized surface plasmon resonance in mid-infra-red and are ideal platforms for surface enhanced infra-red absorption (SEIRA). We also demonstrate a way to design and fabricate complicated patterns by tuning parameters in each exposure step. This method is both high-throughput and low-cost, which is a powerful tool for future infra-red metamaterials applications.

M etamaterials (MMs) are artificial materials which consist of sub-wavelength elements and their spatially averaged response can be recognized as a homogeneous medium with a characteristic effective permittivity $(\varepsilon)$ and permeability $(\mu)^{1,2}$. These man-made materials demonstrate optical properties that are not found in nature and make possible for some exotic applications, such as invisible cloak ${ }^{3}$ and perfect lenses ${ }^{4}$. Recently, visible MMs have been the focus for researches worldwide ${ }^{5-7}$, but the progress was severely limited due to the difficulty in nanofabrication. In contrast, infra-red (IR) MMs are much easier to be fabricated and have been leading to novel applications, such as perfect absorbers $(\mathrm{PAs})^{8-12}$ and electromagnetically induced transparency (EIT) ${ }^{13,14}$. These IR MMs can be used as high efficient thermal absorbers/emitters for thermophotovoltiac cells $^{15}$ or sensitive refractive index sensors for biosensors ${ }^{11,14,16}$. Meanwhile, asymmetric nano-cross arrays have also been reported to work as quarter wave monopole antenna elements which are compatible with chip-based fabrication techniques ${ }^{17}$. In recent years, a new class of metamaterials, known as planar chiral metamaterials (PCMs), has invoked much interest in the scientific community due to their large optical activities and polarization conversion can be achieved in a PCM structure with a thickness at only a fraction of a wavelength ${ }^{18-21}$.

When illuminated the metal nanoparticles with resonant light that matches their localized surface plasmon resonance (LSPR), strong electric field occurs near the surface and is useful to boost the Raman scattering signal for ultrasensitive surface-enhanced Raman scattering (SERS) measurements ${ }^{22}$ and the absorption signal from the vibrational modes of molecules for surface-enhanced IR absorption (SEIRA) spectroscopy. Both techniques can reveal the vibrational fingerprints of bio-molecules. However, the sensitivity is severely limited due to the small intrinsic absorption cross-sections. SEIRA is usually performed with chemically prepared or roughened metal surfaces ${ }^{23,24}$ and its enhancement factor is limited to $10-100$ because of the random distributions of the surface roughness. Recently, SEIRA has been demonstrated from engineered metal nanostructures, such as nano-shells ${ }^{25}$, nano-crescents ${ }^{26}$, and nano-bars ${ }^{27,28}$. These nanostructures exhibit uniform size distributions and can be arranged periodically on top of a surface. These periodic nanostructures can easily achieve a SEIRA signal enhancement of $10^{4}$, which is capable to detect the vibrational signals from a single protein monolayer.

LSPR of an individual nanoparticle can also be tuned by varying the size and shape of the nanoparticle ${ }^{29-33}$. Nanoparticles with more complex shapes, such as nano-crescents ${ }^{34}$ and nano-crosses ${ }^{35,36}$, have also been investigated for various LSPR applications. These complex nanostructures can be precisely fabricated using focusedion-beam milling (FIB) or electron-beam lithography (EBL). However, the low throughput of these techniques is not ideal to fabricate samples with sizes as large as $1 \mathrm{~cm}^{2}$ that is typical for IR absorption spectroscopy. Nanofabrication methods with high-throughput capability, such as interference lithography ${ }^{37-40}$, hole-mask colloidal lithography ${ }^{41}$ and nano-imprint lithography ${ }^{42}$, are the preferred nanofabrication techniques. Furthermore, fabrication of nanoscale triangle arrays have been reported using Nano-sphere Lithography ${ }^{43}$. Micro-spheres have been reported as focusing lenses to focus the incident laser light and ablate the underlining 

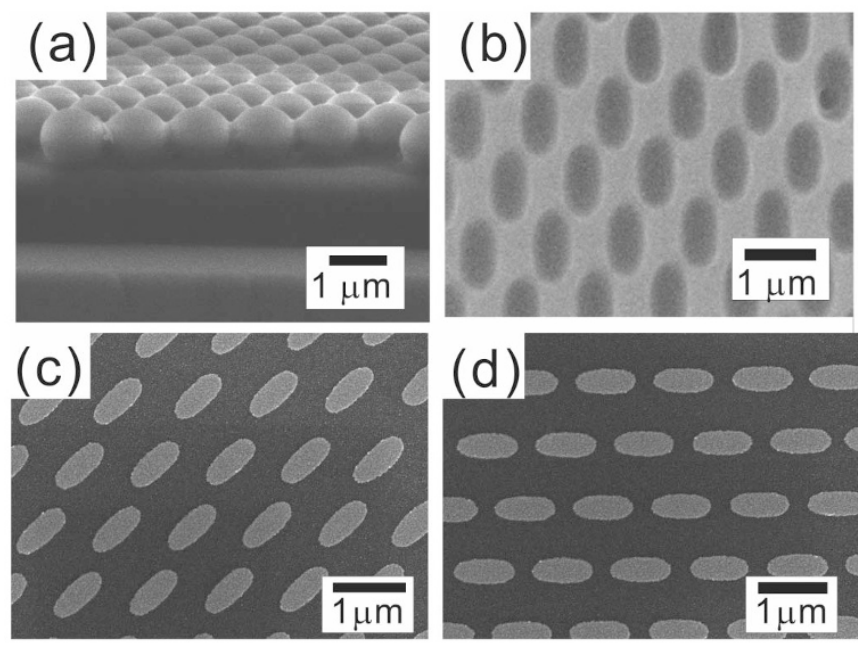

Figure 1 SEM images of (a) the nanosphere array on top of photoresist, (b) the elliptical photoresist hole arrays. (c) and (d) the fabricated nanoellipse arrays with the long-axis pointing to two different directions.

materials ${ }^{44-47}$. These spheres can also be used as phase-shifting lithography masks to fabricate two-dimensional nanostructures ${ }^{31,33,48,49}$. In our previous reports, large-scale nanodisk arrays are successfully fabricated using this method, which we referred it as "NanosphericalLens Lithography (NLL)" 50,51 . Furthermore, the micro-/nanospheres were also reported to be used for micro-/nano- fabrication and imaging ${ }^{52,53}$.

Here we demonstrate the fabrication of IR MMs and PCMs using NLL simply by replacing the light source with a hand-held ultraviolet lamp. The fabricated nano-ellipse arrays are perfect platforms for SEIRA. More complicated IR MMs and PCMs are also demonstrated by precisely controlling the multiple exposure sequences. The proposed method is possible for large-scale fabrication with very low fabrication cost, which should be beneficial for future industrialization of IR MMs and PCMs.

\section{Results}

Nano-ellipse arrays fabricated by replacing the light source of Nanospherical-Lens Lithography with a hand-held UV lamp. The first step for the NLL is to align a single-layered nanosphere array on top of a photoresist (PR) thin film, as illustrated in the scanning electron microscope (SEM) image in Fig. 1(a). The subsequent UV exposure is usually done with a commercial UV aligner as the light source and round-shaped PR hole patterns reveal after PR development ${ }^{50}$. Although commercial UV aligners are standard instruments in semiconductor factories, they are actually quite expensive. Therefore, we have tried to replace it with a hand-held UV lamp, which is cheaper and easily available. To our surprise, elliptical PR hole arrays reveal after PR develop as shown in Fig. 1(b). The diameter of spheres is $1 \mu \mathrm{m}$. By controlling the orientation of the lamp, nano-ellipse arrays with different configurations can be fabricated, as shown in Figs. 1(c) and (d). The long axis of the fabricated nano-ellipse is always parallel to the lamp direction.

In order to explain why elliptical patterns forms when the UV lamp is used as the light source, the light intensity distribution of the UV lamp is measured and shown in Figs. 2(d) and (e). UV lamp is positioned at a height of $\mathrm{z}=15 \mathrm{~cm}$ and oriented along the $\mathrm{y}$-axis. The light intensity is recorded every $1 \mathrm{~cm}$ along all three axes. It is very clear that the light distribution across the xz-plane shown in Fig. 2(d) is very similar to a regular point source while the distribution across the yz-plane shown in Fig. 2(e) is an uniform light source. The results are consistent with the fact that the back reflector exists in a UV lamp and the light is coming out of it with two different propagating behaviors. In order to further illustrate why these elliptical PR holes form, theoretical simulations were performed using a three-dimensional finite-difference time-domain (3DFDTD) method. Figs. 2(a)-(c) illustrate the simulated field energy distributions across the $\mathrm{xz}-$, $y z-$, and xy-planes near a nanosphere at a diameter of $1 \mu \mathrm{m}$. The UV light $(\lambda=365 \mathrm{~nm})$ is emitting from a line-shaped source that is parallel to the $y$-axis. The propagation of the incident light across the $\mathrm{xz}$ - and yz-planes demonstrates different behaviors, which are clearly visible in Figs. 2(a) and (b). The difference in the propagation behavior between the xz- and yz-planes results in different focusing spot sizes between two different polarizations. Fig. 2(c) illustrates the simulated field energy distribution across the $\mathrm{z}=3.2 \mu \mathrm{m}$ plane, which are also marked as white dash lines in Figures 2(a) and 2(b). The field energy distribution is an elliptical-shaped pattern, which explains why elliptical PR holes are formed.

Tunable size and aspect ratio of nano-ellipses. The fabricated nano-ellipses using spheres at a diameter of $1 \mu \mathrm{m}$ are too large to exhibit a surface plasmon resonance in the visible light region, smaller nano-ellipses with smaller periodicity are necessary. How ever, it surprised us that only round PR holes were fabricated when the nanospheres at a diameter of $500 \mathrm{~nm}$ are used. There fore, the aspect ratio between the lengths of major and minor axes strongly depends on the diameter of the nanospheres. Micro/Nanospheres with different diameters are subsequently investigated and we have discovered that the aspect ratio is strongly affected by the diameters of the spheres. As illustrated in Fig. 3(a), the aspect ratio increases to around 3 when the diameter of the sphere increases to $2 \mu \mathrm{m} .5$ different exposure durations are investigated for each size and the corresponding ratio is illustrated as a bar in Fig. 3(a). For each size of sphere, the column located at the right represents a longer exposure time. Figs. 3(b) and (c) represent the lengths of the major and minor axes of the fabricated nano-ellipses using spheres with different diameters. For each size of sphere, the data point at higher location represents a longer exposure duration time. In these figures, we have demonstrated that the length and the aspect ratio of the fabricated nano-ellipses can be tuned by controlling the exposure time and the diameters of spheres. The ratio is tunable be tween 1 and 3 and the length of the major axis is tunable between $200 \mathrm{~nm}$ and $1.4 \mu \mathrm{m}$.

Nano-ellipse array as ideal platforms for SEIRA. One of the major applications for the fabricated nano-ellipse arrays are to be used as platforms for SEIRA. By fabricating the nano-ellipses with their long axis around $1.2 \mu \mathrm{m}$, the LSPR of the major axis is between $1000 \mathrm{~cm}^{-1}$ to $2000 \mathrm{~cm}^{-1}$. This spectral range covers most of the vibrational energy spectra of many chemical and biological species. SEIRA within this spectra range exhibits high potential for ultra-sensitive biosensing capabilities. Fig. 4(a) illustrates the LSPR of the nanoellipse arrays with different lengths of major axis. The periodicity is $1.5 \mu \mathrm{m}$. The LSPR shifts to a lower energy when the length of the major axis becomes smaller. The LSPR peaks are also affected by the periodicity, which is located near $2200 \mathrm{~cm}^{-1}$. In Fig. 4(b), we fix the length of the major axis at $1.1 \mu \mathrm{m}$ and vary the periodicity. A shift to lower energy is observed when using a larger periodicity. A nano-ellipse should exhibit a polarization-dependent optical property. Fig. 4(c) illustrates the polarization-dependent extinction spectra of the fabricated Au nano-ellipse arrays. The periodicity and the length of the major axis are 1.5 and $1.15 \mu \mathrm{m}$, respectively. The polarization-dependent absorbance of the fabricated arrays further verifies the observed extinction response originates from the LSPR of the nano-ellipse arrays. Therefore, we have demonstrated the ability to perform precise tuning of the nano-ellipse arrays for a specific infrared band. By using the polarization-dependent FTIR measurement, we can maximize the FTIR signal to improve the signal intensity of SEIRA. 

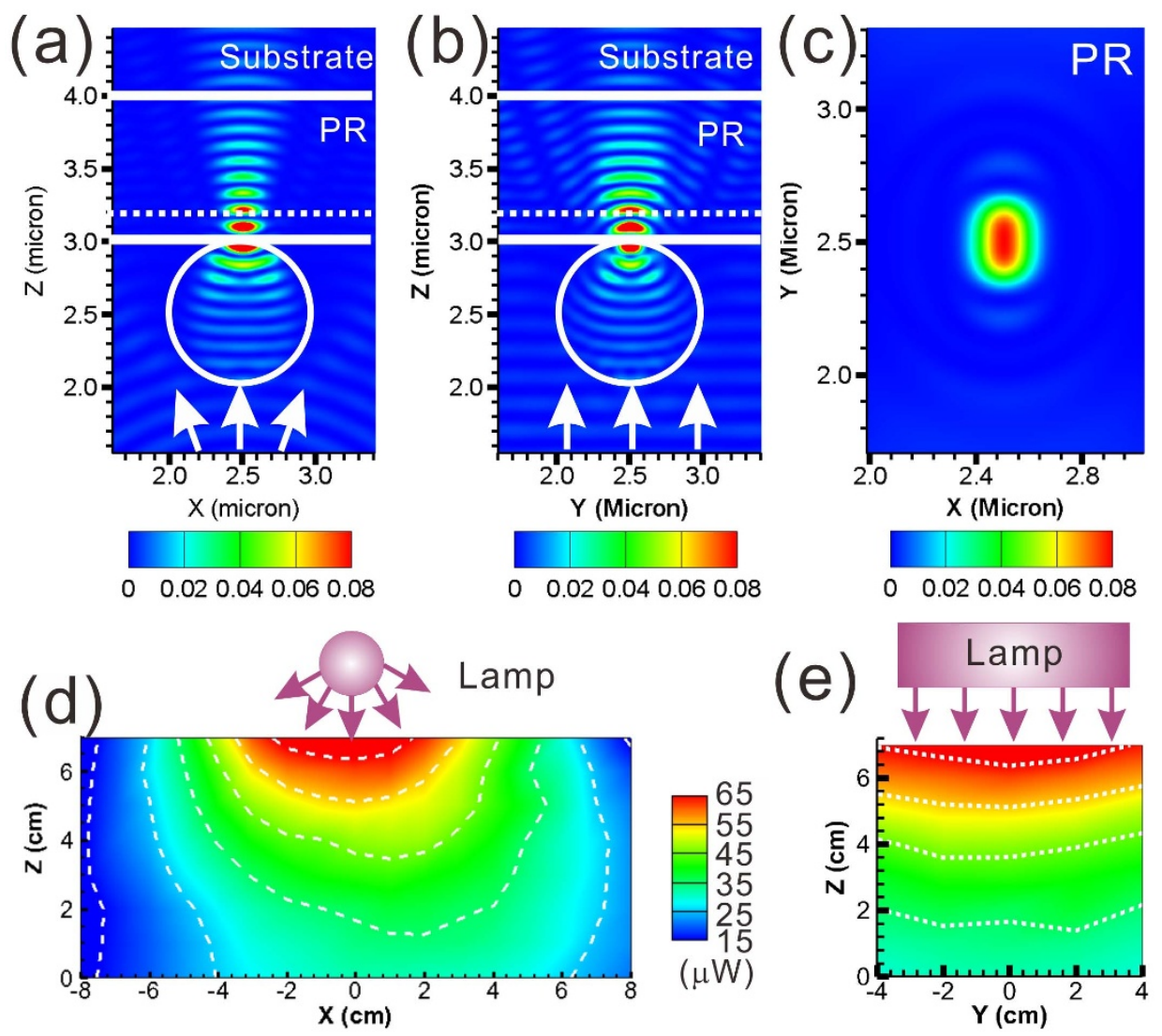

Figure $2 \mid$ Simulated field energy distributions across the (a) xz-plane and (b) yz-plane that demonstrates two different focusing behaviors of the incident light when using UV lamp as the light source. The field energy distribution across the xy-plane $(\mathrm{z}=3.2 \mu \mathrm{m})$ is shown in (c). The dash lines in (a) and (b) indicate the location of $\mathrm{z}=3.2 \mu \mathrm{m}$ plane. Measured light intensity distribution of the UV lamp across the (d) xz-plane and (b) yz-plane. The UV lamp is positioned at $\mathrm{z}=15 \mathrm{~cm}$ and orientated along the $\mathrm{y}$-axis.

Complex IR and chiral metamaterials fabricated with different multiple exposures. In addition to the nano-ellipse arrays, the proposed method can be used to fabricate more complex-shaped metamaterials after multiple exposures. Fig. 5(a) illustrates the experimental configuration for the multiple exposures. The UV lamp is positioned at a constant height of $15 \mathrm{~cm}$ above the sample. The sample can be rotated or shifted along the direction perpendicular to the lamp direction, where $S_{x}$ denotes the shifting distance. The sample shift causes the UV light to incident the surface at an angle. Fig. 5(b) illustrates the simulated field energy distribution across the xz-plane near a nanosphere at a diameter of $1 \mu \mathrm{m}$ when the UV light $(\lambda=365 \mathrm{~nm})$ is incident to the surface at an angle of $30^{\circ}$. The field energy distribution reveals that the incident light exposes the underneath photoresist with an offset center position, which is useful to fabricate complicated metamaterials when exposing with a line-shaped UV lamp.

Fig. 6 illustrates the scanning electron microscopy (SEM) images of different shapes of structures that can be fabricated using the proposed method. Fig. 6(a1) demonstrates a cross array fabricated by performing the $2^{\text {nd }} \mathrm{UV}$ exposure after rotating the lamp for $90^{\circ}$. The durations for both exposures are $100 \mathrm{~s}$ and the resulting cross structures exhibit two legs with similar length. The length for each leg can be separately controlled by changing the duration for each UV exposure. Figs. 6(a2) and (a3) demonstrate two un-even cross arrays which exhibit two different leg lengths. In both figures, the $1^{\text {st }}$ exposure for both samples are kept the same at $100 \mathrm{~s}$ and the $2^{\text {nd }}$ exposure is reduced to $80 \mathrm{~s}$ and $60 \mathrm{~s}$, respectively. It is possible to fabricate a cross structure with an offset center position. This can be simply done by changing the sample position and then performing the $2^{\text {nd }}$ exposure. The durations for the first and second exposures for all the
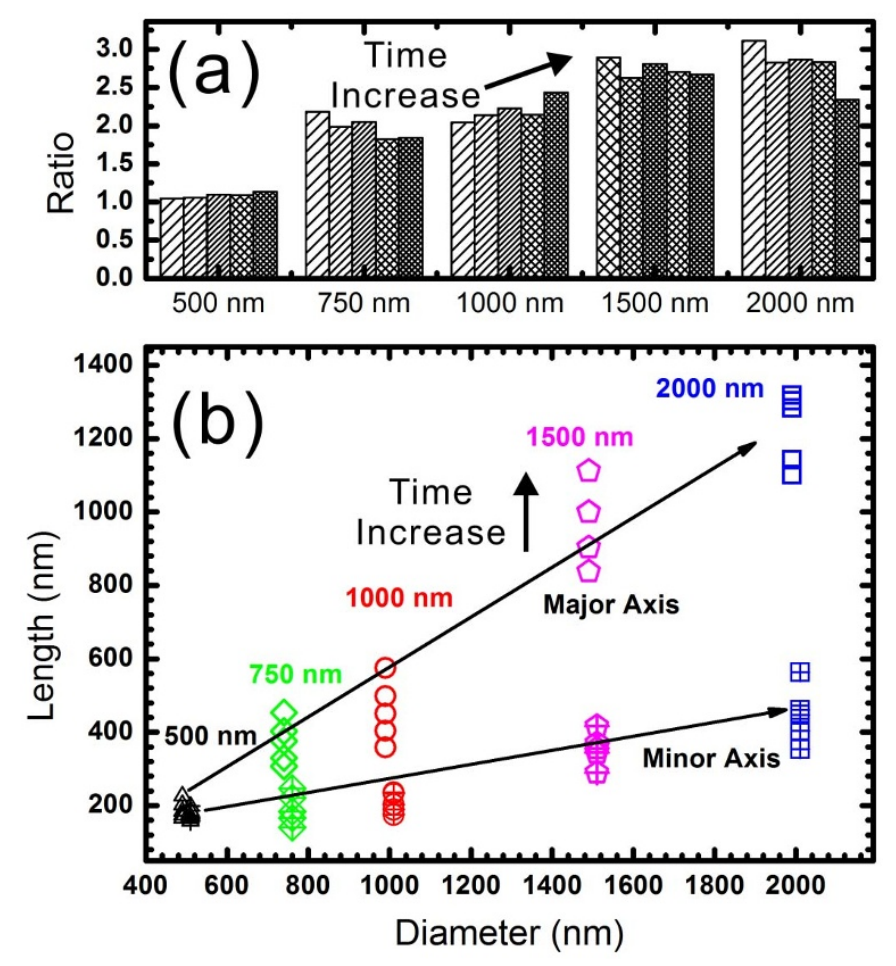

Figure $3 \mid$ (a) Aspect ratio and (b) major and minor axis lengths of the fabricated nano-ellipses using nanospheres at various diameters and different exposure time. 

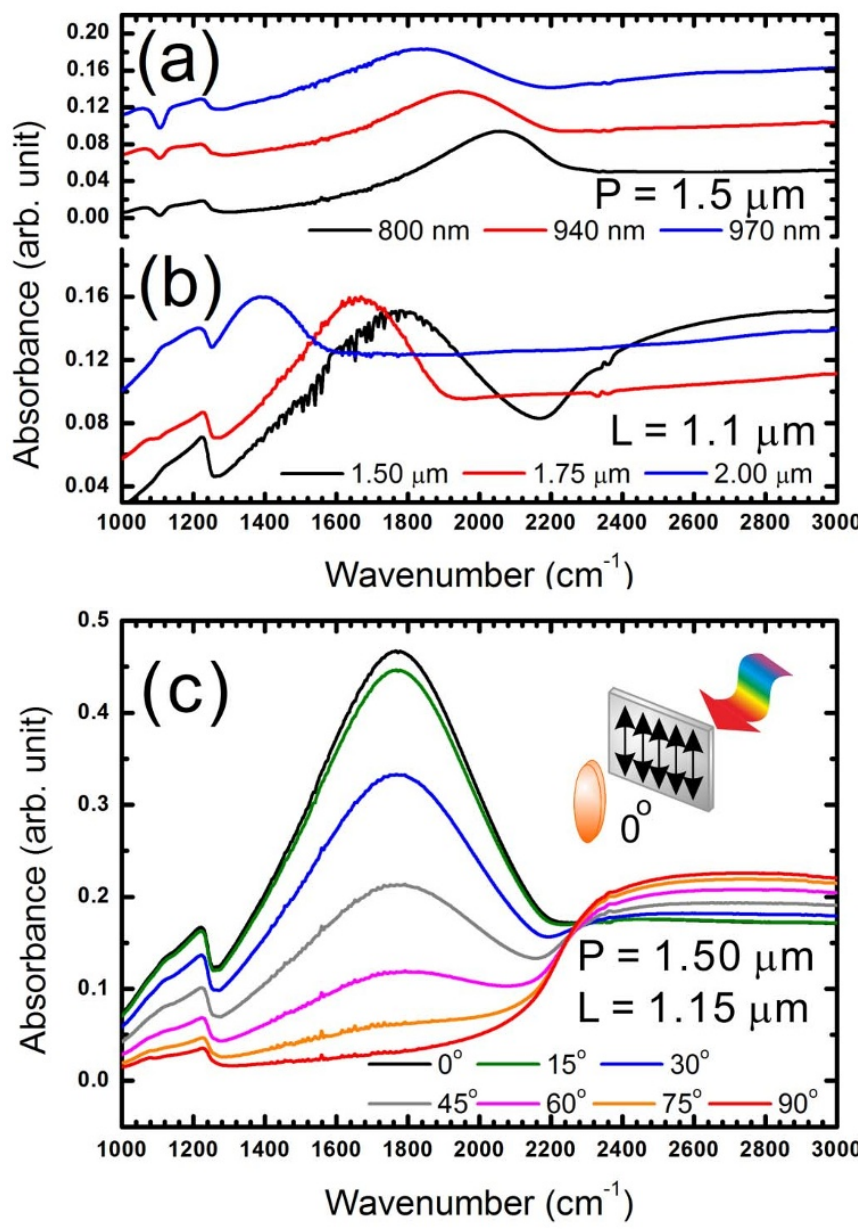

Figure $4 \mid$ FTIR spectra of Au nanoellipse arrays with varying (a) length for long axis and (b) periodicity. (c) Polarization-dependent FTIR spectra of nanoellipse arrays which long-axis length is $1.15 \mathrm{~mm}$ and periodicity is $1.5 \mathrm{~mm}$. Inset in (c) illustrates that $0^{\circ}$ corresponds to the polarizer is aligned along the long-axis of the nanoellipses. samples are set as 100 s. Figs. 6(b1) to (b2) illustrated the SEM images of the resulting cross arrays. The shift of the second leg is clearly observed in each image and the shift becomes larger when $S_{x}$ becomes larger. The shift is about $140 \mathrm{~nm}$ when $S_{x}$ is $3 \mathrm{~cm}$ and increases to $450 \mathrm{~nm}$ when $S_{x}$ is $7.5 \mathrm{~cm}$. It should also be noted that the length of the second leg becomes shorter as the shift of sample location becomes larger, which is reasonable since the sample is further away from the lamp.

It is also possible to fabricate more complicated nanostructures by changing the angle between exposures and the amount of exposure steps taken. Fig. 6(c1) illustrates the SEM image of the nano-ellipse array after a single exposure for $100 \mathrm{~s}$. When performing the $2^{\text {nd }}$ exposure, the sample is clock wisely rotated by only $60^{\circ}$, results in a butterfly array shown in Fig. 6(c2). By rotating the sample again by another $60^{\circ}$, the star array shown in Fig. 6(c3) is fabricated. The duration of the $2^{\text {nd }}$ and $3^{\text {rd }}$ exposures are both kept at $100 \mathrm{~s}$. It is also possible to perform the multiple exposures after shifting the sample location and rotating the sample. In an attempt to preciously describe the exposure parameters, a notation is introduced. For each exposure, a set of parameters are combined in the following manner $\mathrm{E}_{\mathrm{X}}(\mathrm{S} \mathrm{x}$, Angle, Duration), where " $\mathrm{X}$ " indicates the exposure number, " $\mathrm{S}_{\mathrm{x}}$ " the shift distance, "Angle" the angle between the sample and the lamp, and "Duration" the exposure duration. For example, to fabricate a T-shaped element arrays shown in Fig. 6(d1). The exposure sequence is $\mathrm{E}_{1}\left(0 \mathrm{~cm}, 0^{\circ}, 100 \mathrm{~s}\right) / \mathrm{E}_{2}\left(7.5 \mathrm{~cm}, 90^{\circ}, 360 \mathrm{~s}\right)$. The $\mathrm{H}$-shaped elements as shown in Fig. 6(d2) can be fabricated via the following exposure sequence: $\mathrm{E}_{1}\left(0 \mathrm{~cm}, 0^{\circ}, 100 \mathrm{~s}\right) / \mathrm{E}_{2}\left(7.5 \mathrm{~cm}, 90^{\circ}, 300 \mathrm{~s}\right) /$ $\mathrm{E}_{3}\left(7.5 \mathrm{~cm},-90^{\circ}, 300 \mathrm{~s}\right)$. To fabricate U-shaped element arrays shown in Fig. 6(d3), we can perform the following three exposures: $\mathrm{E}_{1}\left(6 \mathrm{~cm}, 0^{\circ}, 300 \mathrm{~s}\right) / \mathrm{E}_{2}\left(6 \mathrm{~cm}, 90^{\circ}, 300 \mathrm{~s}\right) / \mathrm{E}_{3}\left(6 \mathrm{~cm},-90^{\circ}, 300 \mathrm{~s}\right)$.

It is also possible to fabricate PCMs simply by tweaking the exposure sequences, as shown in Figs. 6(e1)-(e4). PCMs consist "flat" chiral elements possessing no line of symmetry in the plane. A planar object is said to be chiral if it cannot be brought to congruence with its mirror image unless it is lifted from the plane ${ }^{18}$. For example, the Lshaped element array shown in Fig. 6(e1) is fabricated after double exposures: $\mathrm{E}_{1}\left(7 \mathrm{~cm}, 0^{\circ}, 300 \mathrm{~s}\right) / \mathrm{E}_{2}\left(7 \mathrm{~cm}, 90^{\circ}, 150 \mathrm{~s}\right)$. If the sample is rotated for $-90^{\circ}$ and the same $2^{\text {nd }}$ exposure is performed, an inverse L-shaped element array appears as shown in Fig. 6(e2). Z-shaped element array is also a type of PCMs as shown in Fig. 6(e3). It can be fabricated by performing the triple exposures: $\mathrm{E}_{1}\left(0 \mathrm{~cm}, 45^{\circ}, 100 \mathrm{~s}\right) /$ $\mathrm{E}_{2}\left(7 \mathrm{~cm}, 90^{\circ}, 300 \mathrm{~s}\right) / \mathrm{E}_{3}\left(7 \mathrm{~cm},-90^{\circ}, 300 \mathrm{~s}\right)$. The mirror image of $\mathrm{Z}$
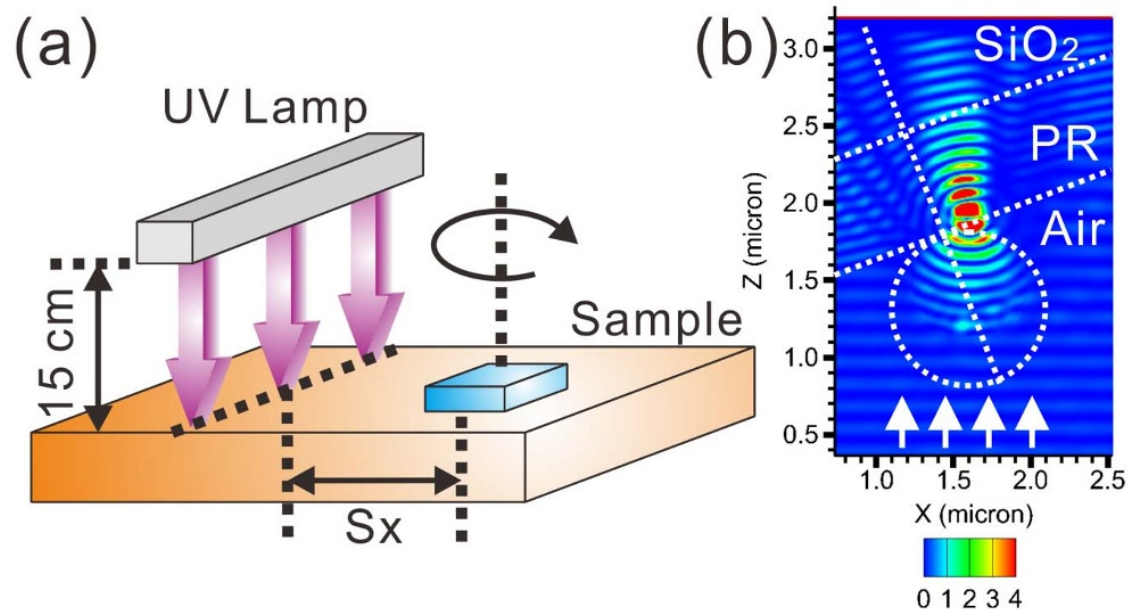

Figure $5 \mid$ (a) Schematical illustration of multiple exposures. The vertical distance between UV lamp and the sample is kept at $15 \mathrm{~cm}$. The sample can be rotated and shifted along one direction. Each exposure is denoted by 3 parameters. $E_{X}\left(S_{x}\right.$, Angle, Duration). "X" denotes the exposure number, " $\mathrm{S}_{\mathrm{x}}$ " the shifting distance, "Angle" the angle between the sample and the lamp, and "Duration" the exposure duration. Multiple exposures after different rotating angles at different $S_{x}$ can produce different types of metamaterials. (b) Simulated field energy distributions across the xz-plane when exposure the samples at a shifted location. The light is focused at an offset center location. 


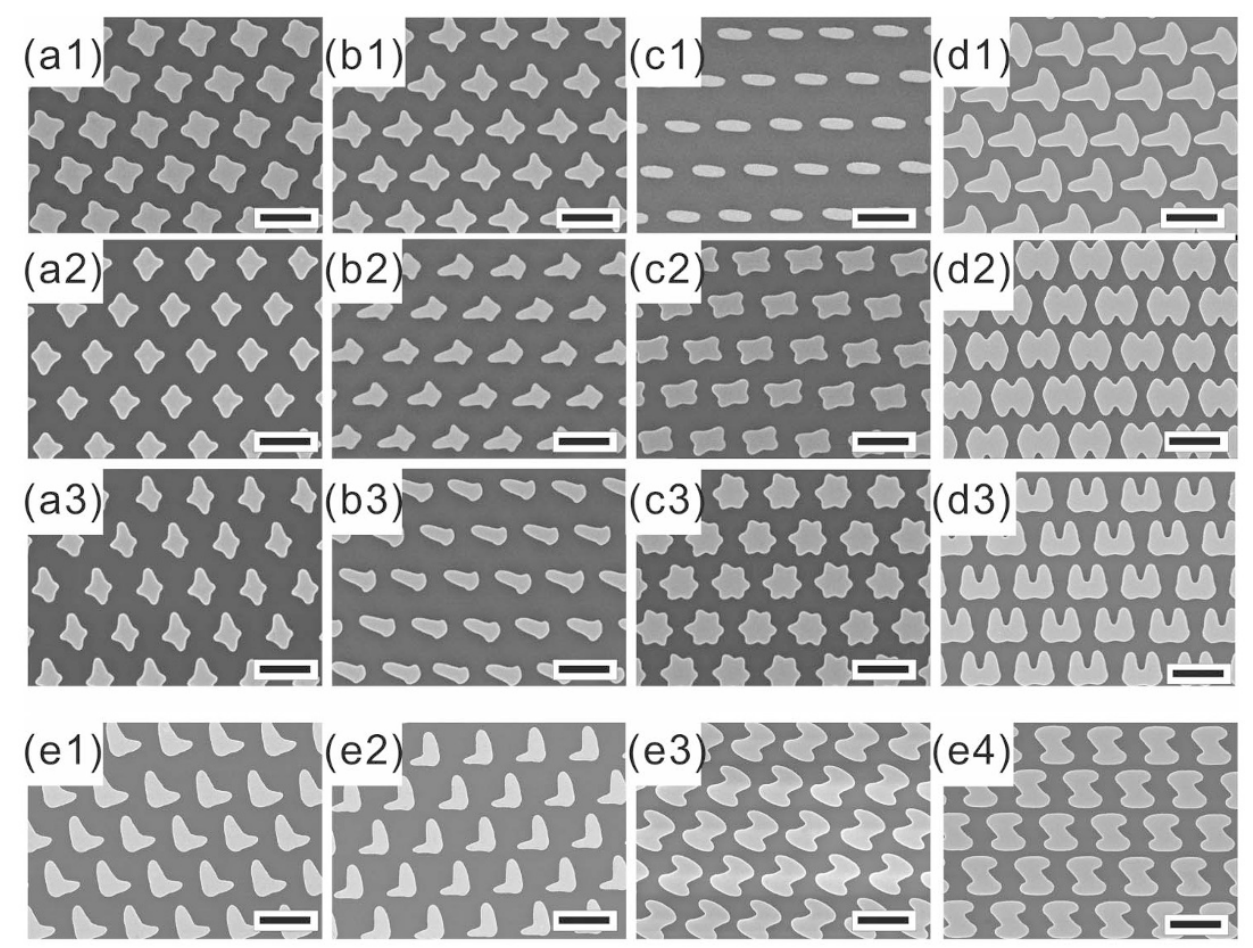

Figure 6 SEM images of the nanostructures fabricated. (a1) to (a3) all with $\mathrm{E}_{1}\left(0 \mathrm{~cm}, 0^{\circ}, 100 \mathrm{~s}\right)$ and varying exposure duration so $\mathrm{E}_{2}\left(0 \mathrm{~cm}, 90^{\circ}, 100 \mathrm{~s}\right)$, $\mathrm{E}_{2}\left(0 \mathrm{~cm}, 90^{\circ}, 80 \mathrm{~s}\right), \mathrm{E}_{2}\left(0 \mathrm{~cm}, 90^{\circ}, 60 \mathrm{~s}\right)$, respectively. (b1) to (b3) are results after $\mathrm{E}_{1}\left(0 \mathrm{~cm}, 0^{\circ}, 100 \mathrm{~s}\right)$ and varying the shift distance $\left(\mathrm{S}_{\mathrm{x}}\right) \mathrm{so} \mathrm{E}_{2}\left(3 \mathrm{~cm}, 90^{\circ}\right.$, $100 \mathrm{~s}), \mathrm{E}_{2}\left(6 \mathrm{~cm}, 90^{\circ}, 100 \mathrm{~s}\right), \mathrm{E}_{2}\left(7.5 \mathrm{~cm}, 90^{\circ}, 100 \mathrm{~s}\right)$, respectively. (c1) to (c3) are results after single, double, and triple exposures by rotating the lamp $60^{\circ}$ after each exposure. The exposure durations are all $100 \mathrm{~s} .(\mathrm{d} 1) \mathrm{E}_{1}\left(0 \mathrm{~cm}, 0^{\circ}, 100 \mathrm{~s}\right) / \mathrm{E}_{2}\left(7.5 \mathrm{~cm}, 90^{\circ}, 360 \mathrm{~s}\right)(\mathrm{d} 2) \mathrm{E}_{1}\left(0 \mathrm{~cm}, 0^{\circ}, 100 \mathrm{~s}\right) / \mathrm{E}_{2}\left(7.5 \mathrm{~cm}, 90^{\circ}, 300 \mathrm{~s}\right) /$ $\mathrm{E}_{3}\left(7.5 \mathrm{~cm},-90^{\circ}, 360 \mathrm{~s}\right)(\mathrm{d} 3) \mathrm{E}_{1}\left(6 \mathrm{~cm}, 0^{\circ}, 300 \mathrm{~s}\right) / \mathrm{E}_{2}\left(6 \mathrm{~cm}, 90^{\circ}, 300 \mathrm{~s}\right) / \mathrm{E}_{3}\left(6 \mathrm{~cm}, 180^{\circ}, 300 \mathrm{~s}\right)(\mathrm{e} 1)$ to (e4) are possible planar chiral metamaterials that can be fabricated. (e1) and (e2) $\mathrm{E}_{1}\left(0 \mathrm{~cm}, 0^{\circ}, 150 \mathrm{~s}\right) / \mathrm{E}_{2}\left(7.5 \mathrm{~cm}, \pm 90^{\circ}, 300 \mathrm{~s}\right)$. (e3) and (e4) $\mathrm{E}_{1}\left(0 \mathrm{~cm}, \pm 45^{\circ}, 100 \mathrm{~s}\right) / \mathrm{E}_{2}\left(7 \mathrm{~cm}, 90^{\circ}, 300 \mathrm{~s}\right) / \mathrm{E}_{3}\left(7 \mathrm{~cm},-90^{\circ}\right.$, $300 \mathrm{~s})$. The Au thickness of all the nanostructures is $15 \mathrm{~nm}$ and the scale bar indicates $2 \mu \mathrm{m}$.

as shown in Figure 6(e4), can be fabricated simply by changing the $1^{\text {st }}$ exposure angle from $45^{\circ}$ to $-45^{\circ}$.

\section{Discussion}

SEM images shown in Fig. 6 have clearly demonstrated the uniqueness of this method. In other method such as nano-imprint or nanostencil lithography, it is almost impossible to make such complicated structures by doing the second and third processes due to the difficulty in re-alignment. Usually, a new hard mold is necessary. Any modification, such as the lengths for each leg or the angle between the legs, also requires new mold fabrication. In addition, both nanoimprint or nano-stencil lithography require to use low throughput nanofabrication method to fabricate the necessary hard mold. It is not practical to fabricate such a hard mold that covers an area as large as $1 \mathrm{~cm}^{2}$ due to the extra long fabrication time. Therefore, a more expensive micro-FTIR instrument is usually required to detect IR signal from the fabricated nanostructures. The focusing of the IR beam is also a potential problem if the IR responses from nanostructures are sensitive to the incident angle of IR beam. The fabricated ellipse array using the proposed method can easily cover an area as large as $1 \mathrm{~cm}^{2}$ and is big enough for conventional FTIR instruments, resulting easier and more accurate measurement. In addition, the fabricated ellipse arrays are uniform in size and cover a large area. Ellipse array with similar optical properties can be repeatedly fabricated. These advantages will make this proposed method as an important tool for future industrial applications.

These fabricated structures are very similar to the reported structures that can be applied in various applications. For examples, the un-even cross array demonstrated in Figs. 6(b1) to (b3) is reported to be a key component for dual-band IR PAs ${ }^{12}$. The $\mathrm{H}-$ shaped MMs demonstrated in Fig. 6(d2) were reported to be used for EIT applications ${ }^{13,14}$. The U-shaped MMs demonstrated in Fig. 6(d3) was used as Fano-resonant asymmetric metamaterials to detect protein monolayer ${ }^{16}$. Recently, the L-shaped MMs were also demonstrated to enhance the second harmonic generation (SHG) of light ${ }^{54,55}$. It is possible to fabricate more complex MMs by performing more than three exposures or depositing multiple layers. We believe the proposed method is powerful enough to fabricate most IR MMs and fabricate them economically in a large scale. It should be noted that each of the above MMs and PCMs exhibits interesting optical properties and applications in various research topics. It is not practical to discuss all of them in detail in this research article. Furthermore, the fabrication throughput of this proposed method can be improved by employing the concepts from micro-lens (MLA) array lithography, which removes the contact problem between the substrate and the spheres ${ }^{56,57}$.

In conclusion, we have demonstrated the fabrication of nanoellipse arrays simply using NLL by replacing the light source with a regular UV lamp. The light is propagating differently along the directions parallel and perpendicular to the lamp directions due to the existing of the back reflector. The orientation of the nano-ellipse can be precisely controlled since the long axis is found to be parallel to the lamp direction. We also found out that the aspect ratio of the nanoellipses strongly depends on the diameters of nanospheres. The larger the nanosphere, the ratio becomes larger. Nano-ellipse arrays with the length of major axis between $200 \mathrm{~nm}$ to $1.4 \mu \mathrm{m}$ are successfully demonstrated, with their LSPR response between visible to midinfrared. It is also demonstrated that tuning the periodicity and exposure time can shift the LSPR response to an optimized spectra range for SEIRA. We also demonstrated a methodical ways to design and fabricate various complicated MMs or PCMs simply by precisely controlling the multiple exposures. The versatile and low-cost nanofabrication method, which can also perform high-throughput 
nanofabrication, should pave the way for more industrial applications of IR MMs or PCMs.

\section{Methods}

Highly resistive silicon substrates that were cut into size of $2 \times 2 \mathrm{~cm}^{2}$ were used in this study. After ultrasonically cleaned in isopropyl alcohol, the substrates were rinsed with deionized water and blow-dried by nitrogen. The nanospheres used in this study were polystyrene (PS) spheres with various diameters (Polyscience Inc.). In the beginning, a thin layer of PR was spin-coated on top of the substrate followed by softbaked at $100{ }^{\circ} \mathrm{C}$ for 3 minutes. The estimated thickness of the PR was about $500 \mathrm{~nm}$. A single-layered hexagonal and close-packed micro-/nano-sphere array was subsequently formed via convective self-assembly method ${ }^{58}$. The sphere array serves as a photolithography phase mask for the subsequent exposure of UV light $(\lambda=365 \mathrm{~nm})$ using a commercial UV lamp (Spectroline ENF-280/FE). The exposed-PR thin film was developed and the remaining spheres were removed. Au thin films are thermally evaporated onto the samples and nano-ellipse arrays revealed after the lift-off process. The solvent used for lift-off is acetone. A scanning electron microscope (SEM; JEOL JSM-6340F field emission scanning electron microscope) was used to analyze the corresponding nanostructures after each step. The lengths of major and minor axes of the fabricated nano-ellipses are subsequently analyzed from the SEM images. The UV light intensity is measured by commercial power meter (Ophir NOVA/PD-300UV). Optical absorption spectra of the fabricated arrays were measured using a commercial Fourier-transform Infrared (FTIR) spectrometer (Bruker VERTEX 70) purged with nitrogen gas and equipped with a KRS-5 variable polarizer from Pike, a Deuteratedtriglycine-sulfate (DTGS) detector at a spectral resolution of $4 \mathrm{~cm}^{-1}$. Electromagnetic simulations were performed with the three-dimensional finite-difference timedomain (3D-FDTD) method, using a freely available software package ${ }^{59}$. The plasma frequency and damping constant of the Au are set at $1.32 \times 10^{16} \mathrm{~Hz}$ and $0.68 \times$ $10^{14} \mathrm{~Hz}$, respectively.

1. Smith, D. R., Pendry, J. B. \& Wiltshire, M. C. K. Metamaterials and negative refractive index. Science 305, 788-792 (2004).

2. Shalaev, V. M. Optical negative-index metamaterials. Nat Photonics 1, 41-48 (2007).

3. Schurig, D. et al. Metamaterial electromagnetic cloak at microwave frequencies. Science 314, 977-980 (2006).

4. Pendry, J. B. Negative refraction makes a perfect lens. Phys Rev Lett 85, 3966-3969 (2000).

5. Lezec, H. J., Dionne, J. A. \& Atwater, H. A. Negative refraction at visible frequencies. Science 316, 430-432 (2007).

6. Burgos, S. P., de Waele, R., Polman, A. \& Atwater, H. A. A single-layer wide-angle negative-index metamaterial at visible frequencies. Nat Mater 9, 407-412 (2010).

7. Xiao, S. M., Chettiar, U. K., Kildishev, A. V., Drachev, V. P. \& Shalaev, V. M. Yellow-light negative-index metamaterials. Opt Lett 34, 3478-3480 (2009).

8. Landy, N. I., Sajuyigbe, S., Mock, J. J., Smith, D. R. \& Padilla, W. J. Perfect metamaterial absorber. Phys Rev Lett 100, 207402 (2008).

9. Liu, X. L., Starr, T., Starr, A. F. \& Padilla, W. J. Infrared Spatial and Frequency Selective Metamaterial with Near-Unity Absorbance. Phys Rev Lett 104, 207403 (2010).

10. Jiang, Z. H., Yun, S., Toor, F., Werner, D. H. \& Mayer, T. S. Conformal Dual-Band Near-Perfectly Absorbing Mid-Infrared Metamaterial Coating. Acs Nano 5, 4641-4647 (2011).

11. Liu, N., Mesch, M., Weiss, T., Hentschel, M. \& Giessen, H. Infrared Perfect Absorber and Its Application As Plasmonic Sensor. Nano Lett 10, 2342-2348 (2010).

12. Chen, K., Adato, R. \& Altug, H. Dual-Band Perfect Absorber for Multispectral Plasmon-Enhanced Infrared Spectroscopy. Acs Nano 6, 7998-8006 (2012).

13. Liu, N. et al. Plasmonic analogue of electromagnetically induced transparency at the Drude damping limit. Nat Mater 8, 758-762 (2009).

14. Liu, N. et al. Planar Metamaterial Analogue of Electromagnetically Induced Transparency for Plasmonic Sensing. Nano Lett 10, 1103-1107 (2010).

15. Diem, M., Koschny, T. \& Soukoulis, C. M. Wide-angle perfect absorber/thermal emitter in the terahertz regime. Phys Rev B 79, 033101 (2009).

16. Wu, C. H. et al. Fano-resonant asymmetric metamaterials for ultrasensitive spectroscopy and identification of molecular monolayers. Nat Mater 11, 69-75 (2012).

17. Adato, R., Yanik, A. A. \& Altug, H. On Chip Plasmonic Monopole NanoAntennas and Circuits. Nano Lett 11, 5219-5226 (2011).

18. Papakostas, A. et al. Optical manifestations of planar chirality. Phys Rev Lett $\mathbf{9 0}$, 107404 (2003)

19. Vallius, T., Jefimovs, K., Turunen, J., Vahimaa, P. \& Svirko, Y. Optical activity in subwavelength-period arrays of chiral metallic particles. Appl Phys Lett 83, 234-236 (2003).

20. Kuwata-Gonokami, M. et al. Giant optical activity in quasi-two-dimensional planar nanostructures. Phys Rev Lett 95, 227401 (2005).

21. Fedotov, V. A. et al. Asymmetric propagation of electromagnetic waves through a planar chiral structure. Phys Rev Lett 97, 167401 (2006).

22. Jeanmaire, D. L. \& Vanduyne, R. P. Surface Raman Spectroelectrochemistry. 1. Heterocyclic, Aromatic, and Aliphatic-Amines Adsorbed on Anodized Silver Electrode. J Electroanal Chem 84, 1-20 (1977).
23. Osawa, M. \& Ikeda, M. Surface-Enhanced Infrared-Absorption of ParaNitrobenzoic Acid Deposited on Silver Island Films - Contributions of Electromagnetic and Chemical Mechanisms. J Phys Chem-Us 95, 9914-9919 (1991).

24. Osawa, M. Surface-enhanced infrared absorption. Near-Field Optics and Surface Plasmon Polaritons 81, 163-187 (2001).

25. Kundu, J., Le, F., Nordlander, P. \& Halas, N. J. Surface enhanced infrared absorption (SEIRA) spectroscopy on nanoshell aggregate substrates. Chem Phys Lett 452, 115-119 (2008)

26. Bukasov, R. \& Shumaker-Parry, J. S. Silver Nanocrescents with Infrared Plasmonic Properties As Tunable Substrates for Surface Enhanced Infrared Absorption Spectroscopy. Anal Chem 81, 4531-4535 (2009).

27. Aksu, S. et al. High-Throughput Nanofabrication of Infrared Plasmonic Nanoantenna Arrays for Vibrational Nanospectroscopy. Nano Lett 10, 2511-2518 (2010).

28. Adato, R. et al. Ultra-sensitive vibrational spectroscopy of protein monolayers with plasmonic nanoantenna arrays. P Natl Acad Sci USA 106, 19227-19232 (2009).

29. Haes, A. J. et al. Plasmonic materials for surface-enhanced sensing and spectroscopy. Mrs Bull 30, 368-375 (2005).

30. Sherry, L. J., Jin, R. C., Mirkin, C. A., Schatz, G. C. \& Van Duyne, R. P. Localized surface plasmon resonance spectroscopy of single silver triangular nanoprisms. Nano Lett 6, 2060-2065 (2006).

31. Wu, W., Dey, D., Katsnelson, A., Memis, O. G. \& Mohseni, H. Large areas of periodic nanoholes perforated in multistacked films produced by lift-off. $J$ Vac Sci Technol B 26, 1745-1747 (2008).

32. Neubrech, F. et al. Resonant Plasmonic and Vibrational Coupling in a Tailored Nanoantenna for Infrared Detection. Phys Rev Lett 101, 157403 (2008).

33. Wu, W., Dey, D., Memis, O. G., Katsnelson, A. \& Mohseni, H. Fabrication of large area periodic nanostructures using nanosphere photolithography. Nanoscale Res Lett 3, 351-354 (2008).

34. Bukasov, R. \& Shumaker-Parry, J. S. Highly tunable infrared extinction properties of gold nanocrescents. Nano Lett 7, 1113-1118 (2007).

35. Verellen, N. et al. Plasmon Line Shaping Using Nanocrosses for High Sensitivity Localized Surface Plasmon Resonance Sensing. Nano Lett 11, 391-397 (2011).

36. Rodriguez-Fortuno, F. J. et al. Highly-sensitive chemical detection in the infrared regime using plasmonic gold nanocrosses. Appl Phys Lett 98, 133118 (2011).

37. Feth, N., Enkrich, C., Wegener, M. \& Linden, S. Large-area magnetic metamaterials via compact interference lithography. Opt Express 15, 501-507 (2007).

38. Xie, Q. et al. Fabrication of nanostructures with laser interference lithography. J Alloy Compd 449, 261-264 (2008).

39. Yun, J. et al. Converting carbon nanofibers to carbon nanoneedles: catalyst splitting and reverse motion. Nanoscale 2, 2180-2185 (2010).

40. Choi, W. K. et al. A combined top-down and bottom-up approach for precise placement of metal nanoparticles on silicon. Small 4, 330-333 (2008).

41. Fredriksson, H. et al. Hole-mask colloidal lithography. Adv Mater 19, 4297-4302 (2007).

42. Lee, S. W. et al. Highly Sensitive Biosensing Using Arrays of Plasmonic Au Nanodisks Realized by Nanoimprint Lithography. Acs Nano 5, 897-904 (2011).

43. Hulteen, J. C. \& Vanduyne, R. P. Nanosphere Lithography - a Materials General Fabrication Process for Periodic Particle Array Surfaces. Journal of Vacuum Science \& Technology a-Vacuum Surfaces and Films 13, 1553-1558 (1995).

44. Wang, Z. B. et al. Laser micro/nano fabrication in glass with tunable-focus particle lens array. Opt Express 16, 19706-19711 (2008).

45. Guo, W. et al. Near-field laser parallel nanofabrication of arbitrary-shaped patterns. Appl Phys Lett 90, 243101 (2007).

46. Li, L. et al. Large-area laser nano-texturing with user-defined patterns. J Micromech Microeng 19, 054002 (2009).

47. Pena, A., Wang, Z. B., Whitehead, D. \& Li, L. Direct writing of micro/nano-scale patterns by means of particle lens arrays scanned by a focused diode pumped Nd:YVO4 laser. Appl Phys a-Mater 101, 287-295 (2010).

48. Hou, C. H. et al. Output power enhancement of light-emitting diodes via twodimensional hole arrays generated by a monolayer of microspheres. Appl Phys Lett 95, 133105 (2009).

49. Wu, W., Katsnelson, A., Memis, O. G. \& Mohseni, H. A deep sub-wavelength process for the formation of highly uniform arrays of nanoholes and nanopillars. Nanotechnology 18, 485302 (2007).

50. Chang, Y. C., Wang, S. M., Chung, H. C., Tseng, C. B. \& Chang, S. H. Observation of Absorption-Dominated Bonding Dark Plasmon Mode from Metal-InsulatorMetal Nanodisk Arrays Fabricated by Nanospherical-Lens Lithography. Acs Nano 6, 3390-3396 (2012).

51. Chang, Y. C., Chung, H. C., Lu, S. C. \& Guo, T. F. A large-scale sub-100 nm Au nanodisk array fabricated using nanospherical-lens lithography: a low-cost localized surface plasmon resonance sensor. Nanotechnology 24, 095302 (2013).

52. Chong, T. C., Hong, M. H. \& Shi, L. P. Laser precision engineering: from microfabrication to nanoprocessing. Laser Photonics Rev 4, 123-143 (2010)

53. Wang, Z. B. et al. Optical virtual imaging at $50 \mathrm{~nm}$ lateral resolution with a whitelight nanoscope. Nat Commun 2, 218 (2011).

54. Kauranen, M. \& Zayats, A. V. Nonlinear plasmonics. Nat Photonics 6, 737-748 (2012). 
55. Czaplicki, R. et al. Enhancement of Second-Harmonic Generation from Metal Nanoparticles by Passive Elements. Phys Rev Lett 110, 093902 (2013).

56. Lin, Y. et al. Ultrafast-laser-induced parallel phase-change nanolithography. Appl Phys Lett 89, 041108 (2006).

57. Chen, Z. C., Rahmani, M., Gong, Y. D., Chong, C. T. \& Hong, M. H. Realization of Variable Three-Dimensional Terahertz Metamaterial Tubes for Passive Resonance Tunability. Adv Mater 24, Op143-Op147 (2012).

58. Ormonde, A. D., Hicks, E. C. M., Castillo, J. \& Van Duyne, R. P. Nanosphere lithography: Fabrication of large-area Ag nanoparticle arrays by convective selfassembly and their characterization by scanning UV-visible extinction spectroscopy. Langmuir 20, 6927-6931 (2004).

59. Oskooi, A. F. et al. MEEP: A flexible free-software package for electromagnetic simulations by the FDTD method. Comput Phys Commun 181, 687-702 (2010).

\section{Acknowledgments}

We thank the financial support from National Science Council, Taiwan under Grant Number (NSC 101-2112-M-006-013-MY2). We also thank for the collaboration of Center of Micro/Nano Science and Technology, National Cheng Kung University, Tainan, Taiwan. The National Center for High-Performance Computing of Taiwan and the Computer and
Network of National Cheng Kung University are also acknowledged for uses of high performance computing facilities. The valuable suggestions from Professors Yung-Chiang Lan and Shih-Hui Chang of Department of Photonics are also much appreciated.

\section{Author contributions}

Y.C.C. conceived the design, supervised the whole projects, performed the electromagnetic simulations and wrote the manuscript. S.C.L., H.C.C., S.M.W. fabricated the samples and performed various characterizations. T.D.T. and T.F.G. performed the FTIR measurements. All authors reviewed the manuscript.

\section{Additional information}

Competing financial interests: The authors declare no competing financial interests.

How to cite this article: Chang, Y.-C. et al. High-Throughput Nanofabrication of Infra-red and Chiral Metamaterials using Nanospherical-Lens Lithography. Sci. Rep. 3, 3339; DOI:10.1038/srep03339 (2013).

This work is licensed under a Creative Commons Attribution 3.0 Unported license To view a copy of this license, visit http://creativecommons.org/licenses/by/3.0 